\title{
Driver glance behaviors and scanning patterns: Applying static and dynamic glance measures to the analysis of curve driving with secondary tasks
}

\author{
Heejin Jeong ${ }^{1} \odot \mid$ Ziho Kang ${ }^{2} \mid$ Yili Liu $^{3}$
}

${ }^{1}$ Department of Mechanical and Industrial Engineering, University of Illinois at Chicago, Chicago, Illinois

${ }^{2}$ School of Industrial and Systems Engineering, University of Oklahoma, Norman, Oklahoma

${ }^{3}$ Department of Industrial and Operations Engineering, University of Michigan, Ann Arbor, Michigan

\section{Correspondence}

Heejin Jeong, University of Illinois at Chicago, Chicago, IL.

Email: heejinj@uic.edu

Funding information

Rackham Graduate Student Research Grant from University of Michigan, Grant/Award Number: U051990

\begin{abstract}
Performing secondary tasks (or non-driving-related tasks) while driving on curved roads may be risky and unsafe. The purpose of this study was to explore whether driving safety in situations involving curved roads and secondary tasks can be evaluated using multiple measures of eye movement. We adopted Markov-based transition algorithms (i.e., transition/stationary probabilities, entropy) to quantify drivers' dynamic eye movement patterns, in addition to typical static visual measures, such as frequency and duration of glances. The algorithms were evaluated with data from an experiment (Jeong \& Liu, 2019) involving multiple road curvatures and stimulus-response secondary task types. Drivers were more likely to scan only a few areas of interest with a long duration in sharper curves. Total head-down glance time was longer in less sharp curves in the experiment, but the probability of head-down glances was higher in sharper curves over the long run. The number of reliable transitions between areas of interest varied with the secondary task type. The visual scanning patterns for visually undemanding tasks were as random as those for visually demanding tasks. Markov-based measures of dynamic eye movements provided insights to better understand drivers' underlying mental processes and scanning strategies, compared with typical static measures. The presented methods and results can be useful for in-vehicle systems design and for further analysis of visual scanning patterns in the transportation domain.
\end{abstract}

\section{KEYWORDS}

driver glance, driving safety, Markov chain, visual entropy, visual transition/stationary distribution

\section{1 | INTRODUCTION}

Driving is a visually demanding task that requires continuous attention on multiple objects, including traffic, pedestrians, road signs, and in-vehicle elements (Robinson, Erickson, Thurston, \& Clark, 1972; Sivak, 1996). Many studies have investigated driver glance behavior and found that glance behavior is a key indicator of drivers' underlying cognitive processes and can assist in evaluating driving performance and safety (e.g., Green, 2015; Liang, Lee, \& Yekhshatyan, 2012; Victor et al., 2015).
Previous studies have identified and quantified drivers' glance behavior using multiple glance measures. Most of these glance measures have focused on the glance's static targets or areas of interest (AOIs), such as lead vehicles, rear-view mirrors, and roads, and on the frequency and duration of the glances on the AOIs (e.g., Mourant \& Rockwell, 1970; Werneke \& Vollrath, 2012). As researchers became interested in the risk of distracted driving, attention has been paid to how often or how long drivers look away from the road (i.e., eyes-off-road frequency 
and time). In general, previous distracted driving studies revealed that the risk of vehicle crashes increases when the drivers take their eyes off from the road more often and longer (Liang, Horrey, \& Hoffman, 2015; Sodhi, Reimer, \& Llamazares, 2002). However, these static glance measures have a limitation on evaluating sequences (or transitions) of eye fixation between each $\mathrm{AOI}$ and glance's dynamic characteristics.

Analyzing visual scanning patterns and dynamic characteristics can help in understanding individuals' underlying mental processes and scanning strategies; thus, these analyses can help in minimizing potential accidents and in designing appropriate humanmachine interfaces. To date, although there have been many visual scanning studies using dynamic glance measures (e.g. transitions of eye fixation) in aviation (Harris, Glover, \& Spady, 1986, Haslbeck \& Zhang, 2017, Kang \& Landry, 2014, 2015, Marchitto, Di Stasi, \& Cañas, 2012), relatively few studies have explored drivers' visual scanning patterns and dynamic characteristics. Underwood, Chapman, Brocklehurst, Underwood, and Crundall (2003) identified driver's three scanning patterns (i.e., single/two/three-fixation scanpaths) while driving on different road types (i.e., rural, suburban and dual-carriageway). They used first-order Markov matrices and the transition probabilities within each matrix to quantify and compare sequences of eye fixation between novice and experienced drivers. Bao and Boyle (2009) investigated age difference in driver's visual scanning at intersections, by measuring the time proportion of scanning to three areas (i.e., left, right sides, and rear-view mirror), and visual entropy rate as a measure of randomness in visual scanning. In recent research by Wang, Bao, Du, Ye, \& Sayer (2017), drivers' eye glance patterns were quantified when drivers were engaged in cell phone-related visual-manual tasks. However, the existing literature is not sufficient to determine whether driving safety can be evaluated using both static and dynamic glance measures; moreover, diverse modalities of the secondary task have not been investigated. To address these current research gaps, in this study, we adopt both static and dynamic glance measures to analyze drivers' glance behaviors and scanning patterns in a complex driving scenario, involving curved-road driving with multimodal secondary tasks, as an example. In addition, we aim to explore whether driving safety in the scenarios can be evaluated using the multiple measures of eye movement.

\section{2 | MULTIPLE MEASURES OF EYE MOVEMENT}

In this study, five eye-tracking measures (i.e., two static and three dynamic) were used to identify drivers' glance behaviors and scanning patterns.

First, we used two typical static glance measures that suggested by SAE J2396 (2000) and ISO 15007-1 (2002) to quantify drivers' glance behavior: the number of glances and total glance time at each AOI. The number of glances indicates the count of glances to an AOI during a certain period. The total glance time was defined as the sum of all glance durations to an $\mathrm{AOI}$ during a certain condition.

Additionally, three dynamic glance measures were used as visual scanning measures: visual transition probability, visual stationary probability, and visual entropy. These measures are derived from a simple Markov-based transition algorithm, a stochastic process assuming that each state (in this case, an AOI or a fixation) is dependent only on the previous state. Markov's process has been used as an eye-tracking analytic tool in diverse fields, such as aviation (Allsop \& Gray, 2014; Ellis \& Stark, 1986; Itoh, Hayashi, Tsukui, \& Saito, 1990), business marketing (Liechty, Pieters, \& Wedel, 2003), art appreciation (Krejtz et al., 2015), and health care (e.g., Di Stasi et al., 2016), but few in driving (e.g., Schieber \& Gilland, 2008; Underwood et al., 2003).

\section{1 | Visual transition probability}

The visual transition probability is the probability of eye fixation movement from one state to another in a single step. Figure 1 shows two examples of eye glance transitions between three AOls. Case 1 is normal, and Case 2 shows the maximum randomness of visual scanning (or maximum entropy). The arrows indicate the direction of transition from one $\mathrm{AOI}$ to another $\mathrm{AOI}$, and the numbers represent the frequency of glance transitions between AOls.

Visual transition probability matrices can be created based on visual transitions between AOls, as shown in Table 1.

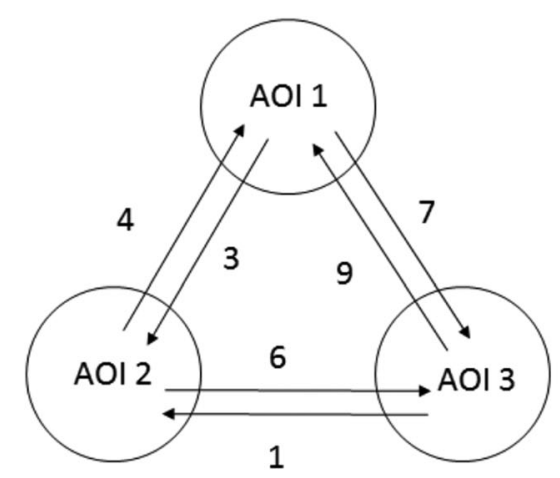

Case 1

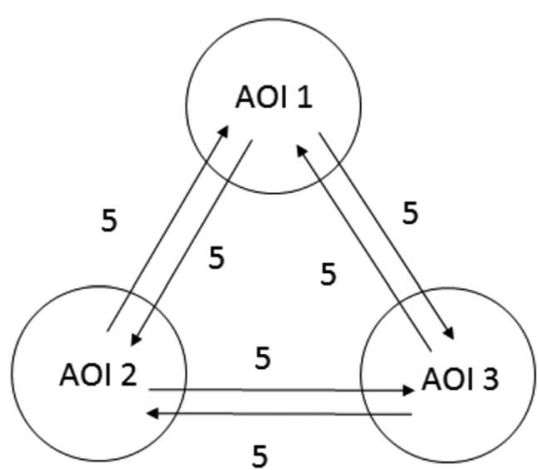

Case 2
FIGURE 1 Examples of visual transitions between three AOls. AOls, areas of interests 
TABLE 1 Examples of the visual transition probability matrix

\begin{tabular}{|c|c|c|c|c|c|c|c|}
\hline From/To & \multicolumn{3}{|l|}{ Case $\mathbf{A}$} & From/To & \multicolumn{3}{|l|}{ Case B } \\
\hline AOI 1 & - & .3 & .7 & AOI 1 & - & .5 & .5 \\
\hline AOI 3 & .1 & .9 & - & AOI 3 & .5 & .5 & - \\
\hline
\end{tabular}

Abbreviations: $\mathrm{AOI}$, areas of interest.

\section{2 | Visual stationary probability}

This is a method for analyzing the long-run properties of Markov chains (Hillier, 2012; Ross, 2014). A typical example of using the stationary probability would be forecasting weather based on the patterns of previous days' weather status, such as predicting the probability of sunny days over the long run. If the number of visual transitions is large enough (or close to infinite), all the rows of the transition matrix will have identical values, so the probability of eye fixation on each AOI no longer depends on the initial AOI. In other words, the visual stationary probability can be used to answer the question, in the long run, what proportion of time will be spent looking at the specific AOls?

Suppose we consider the eye glance transition among $n$ AOls as a Markov chain having state (meaning the $A O I$ in this study) space $S=\{i=1,2, \ldots, n\}$ and transition matrix $P$ is:

$$
\mathrm{P}=\left[\begin{array}{ccc}
p_{11} & \cdots & p_{1 n} \\
\vdots & \ddots & \vdots \\
p_{n 1} & \cdots & p_{n n}
\end{array}\right]
$$

Vector $\pi^{T}$ is a stationary (or an equilibrium) distribution for $P$ when $\pi^{T} P=\pi^{T}, \sum_{i=1}^{n} \pi_{i}=1$, and $\pi_{i} \geq 0$ for all $i$.

The visual stationary probability $\pi_{i}$ can be considered as the probability of total time spent at $i^{\text {th }} \mathrm{AOI}$ once the chain reaches the stationary distribution. For example, in this study, $\pi_{\mathrm{ID}}$ indicates the probability of time spent at the input device (ID), whereas $\pi_{\mathrm{FRV}}$ indicates the probability of time spent at far road view (FRV) in the long run.

Using the examples in Table 1, $0.4 \pi_{2}+.1 \pi_{3}=\pi_{1}, 0.3 \pi_{1}+.9 \pi_{3}=\pi_{2}$, $0.7 \pi_{1}+.6 \pi_{2}=\pi_{3} ; \sum_{i=1}^{3} \pi_{i}=1$ thus, $\pi_{1}=0.20264, \pi_{2}=0.40969$, $\pi_{3}=0.38767$ (for Case A). $0.5 \pi_{2}+0.5 \pi_{3}=\pi_{1}, 0.5 \pi_{1}+0.5 \pi_{3}=\pi_{2}$, $0.5 \pi_{1}+0.5 \pi_{2}=\pi_{3} ; \sum_{i=1}^{3} \pi_{i}=1$ thus, $\pi_{1}=\pi_{2}=\pi_{3}=0.333$ (for Case B). That is, the probabilities that eye glance is fixated at the each AOI is $20.2 \%, 41.0 \%, 38.8 \%$ for Case 1, equally $33.3 \%$ at the all AOls for Case 2 , if the visual scanning patterns continuously repeated.

\section{3 | Visual entropy}

Visual entropy is a measure to quantify complexity or randomness of visual scanning between AOls. It is calculated using the following equation (Allsop \& Gray, 2014; Ellis \& Stark, 1986; Krejtz et al., 2015; Schieber \& Gilland, 2008), based upon Shannon's entropy (Shannon \& Weaver, 1998):

$$
\begin{aligned}
& \text { Visual entropy }=\sum_{i=1}^{n}\left[\sum_{j=1}^{n} Q_{i j}\right] p\left(x_{i}\right), i \neq j \\
& \begin{array}{l}
Q_{i j}=-p\left(y_{i j} \mid x_{i}\right) \log _{2} p\left(y_{i j} \mid x_{i}\right), \text { if } p\left(y_{i j} \mid x_{i}\right)>0 \\
\quad=0 \text {, otherwise, }
\end{array}
\end{aligned}
$$

where $n$ is the number of AOls, $p\left(x_{i}\right)$ is the probability of glance duration on the $i^{\text {th }} \mathrm{AOI}$ among all AOIs, and $p\left(y_{i j} \mid x_{i}\right)$ is the conditional probability of fixating $j^{\text {th }} \mathrm{AOI}$ based upon a current fixation on $i^{\text {th }} \mathrm{AOI}$.

The visual entropy can be calculated from the values in the transition probability matrix. Assumed that the probabilities of time fixating on the each $\mathrm{AOI}$ are equal (for simple calculation, here), or $p\left(x_{1}\right)=p\left(x_{1}\right)=p\left(x_{1}\right)=1 / 3$. Visual entropy $=(-1 /$ 3) $\times\left(0.3 \times \log _{2}(0.3)+0.7 \times \log _{2}(0.7)\right)+(-1 / 3) \times\left(0.4 \times \log _{2}(0.4)+0.6 \times\right.$ $\left.\log _{2}(0.6)\right)+(-1 / 3) \times\left(0.1 \times \log _{2}(0.1)+0.9 \times \log _{2}(0.9)\right)=0.77$ for Case $1,(-1 / 3) \times\left(0.5 \times \log _{2}(0.5)+0.5 \times \log _{2}(0.5)\right) \times 3=1$ for Case 2 . The minimum visual entropy is zero when minimum randomness exists as defined by the eye glances fixated in only single AOI, whether maximum visual entropy is when the transitions from each AOI are equally distributed to all other AOIs (Ellis \& Stark, 1986). However, the maximum is depending on the number of AOls. For the examples in Table 1, the number of AOls was three, so the maximum entropy is 1 . However, in the current study, the visual entropy used in this study is ranging from 0 to 2.58 , because $7 \mathrm{AOls}$ were used.

\section{3 | EXPERIMENT METHOD}

\section{1 | Participants}

Twenty-four subjects (16 males and 8 females; age range, 19-31; $M=22.6, S D=3.53$ ) participated in this experiment. All participants held a valid driver's license and experienced an annual average driving mileage of 24,000 miles. They reported normal or corrected to normal vision. This research was approved by the Institutional Review Board at the University of Michigan. Informed consent was obtained from each participant.

\section{2 | Apparatus}

The experiment was conducted in a fixed-base driving simulator, equipped with a Logitech G27 RT racing wheelset (i.e., a force-feedback 
steering wheel and accelerator/brake pedals) and a 24-inch LCD monitor (HP ZR24w, $1920 \times 1200$ pixels). Eye movements were recorded by an eye-tracker (Gazepoint GP3), positioned between the monitor and the steering wheel. The accuracy of the eye tracker was ranging from $0.5^{\circ}$ to $1^{\circ}$ of visual angle. Both driving and eye tracking data were collected at a rate of $60 \mathrm{~Hz}$. For the secondary tasks while driving, MIT AgeLab NBack App (Reimer et al., 2014) was installed in a 5.7-inch touchscreen device (Samsung Galaxy Note 4; $2560 \times 1440$ pixels) and the device was mounted 12-inch away from the center of the steering wheel in the right direction.

Using the Open Racing Car Simulator, eight driving scenarios were developed, comprising multiple curved rural roads (curvature radii $=100,200,400$, and $800 \mathrm{~m}$ ) and transition straight roads (road length $=300 \mathrm{~m}$ ) between each curved road. The direction of curved roads was equally designed in both left and right. All driving scenarios had a different sequence of curvatures and directions and they were randomly given to the participants. The average driving time for each scenario was approximately 4.5 min with an average driving speed of $50-60 \mathrm{~km} / \mathrm{h}$.

\section{3 | Driving and stimulus-response tasks}

Participants were instructed to control a simulated vehicle as close as possible to the center of the driving lane. There were no other vehicles in the driving scenario. While driving, they were asked to perform a stimulus-response (S-R) task using an n-back application software. The n-back task was originally developed as a method to evaluate human's working memory by receiving a sequence of stimuli and responding to the one from $n$-steps ( $n=0,1,2$, etc.) earlier in the sequence. Of the $n$ back tasks, only the 0-back task (the easiest level; responding to the stimulus "just" received, also called "digit repetition task") was used in this study as a secondary task, to primarily focus on comparing the effects of four different S-R types on glance behavior, rather than comparing cognitive workload from different type of n-back levels.

The task contained two different stimulus types (i.e., visual and auditory) and two different response types (i.e., manual and speech). The visual stimuli consisted of ten Arabic numerals (i.e., 0-9) and the auditory stimuli were the corresponding voice sounds of the ten numerals. Each stimulus was presented every $2.25 \mathrm{~s}$ with a $0.75 \mathrm{~s}$ gap between each stimulus. A hundred of visual or auditory stimuli were presented for $5 \mathrm{~min}$ in each session. While driving, participants were asked to click the button (as the manual response) or repeat aloud (as the speech response) corresponding to the number that was "just" heard from the speaker (as the auditory stimulus) or presented on the display (as the visual stimulus). Before each session, participants were instructed to respond to each stimulus as accurately and quickly as possible, but to prioritize the safe driving over than secondary task performance.

\section{4 | Experimental procedure}

Upon arrival at the laboratory, all participants were informed about the purpose and contents of the experiment. After they conducted a 5 to 10-min practice drive to become familiar with both driving and secondary tasks, the calibration for the eye-tracking system was conducted. All 24 participants performed four sessions that each session has each stimulusresponse task type while driving on all levels of curves. The sequence of the four sessions was balanced across participants to mitigate the learning effects of their driving behaviors with the four different secondary tasks. Note that different sequence of driving curvatures and directions were randomly given to the participants in each drive, as mentioned in the Apparatus section. Each session took ranging from 5 to $7 \mathrm{~min}$, depending on participants' driving speed. Between the sessions, they had a 5 -min break to minimize the effect of fatigue from the previous to the next session. For additional details of the experimental tasks and procedure, see Jeong and Liu (2019).

\section{$3.5 \quad$ Areas of interest}

The definitions of seven AOls (predetermined areas within the visual scene) are shown in Figure 2, from left-up to right-down, side scenery view (SSV), rear-view mirror (RVM), far scenery view (FSV), far road view (FRV), near road view (NRV), instrument panel (IP), and input device (ID).

\section{6 | Data analysis}

In the current study, a $2 \times 4$ analysis was used, with driving road curvature (Sharp [curvature radius $=100 \mathrm{~m}$ ] vs. Moderate [800 $\mathrm{m}$ ]; note that only the biggest and smallest curvature radii were selected to investigate the effect of the road curvatures on driver eye movement) and the stimulus-response task type (auditory-manual [A-M], auditory-speech [A-S], visual-manual [V-M], and visual-speech [V-S]). All eye fixation data obtained while driving on the moderate curve were divided by a value of 8 to make the identical condition with the data while driving on the sharp curve, based on the assumption that it takes eight times longer while driving on the moderate curve than the sharp curve. The normality tests for all dependent variables were done using the Shapiro-Wilk test. For nonparametric data including the two static glance measures, visual entropy, and driving speed, the Mann-Whitney $U$ test (for comparison between the two curvatures) and Kruskal-Wallis $\mathrm{H}$ test (for comparison between the 4 secondary task types) were conducted. Stationary probability data were analyzed using the repeated-measures two-way analysis of variance (ANOVA) with 2 curvatures $\times 4$ secondary task types. The Levene's test for homogeneity of variance was used to investigate the assumption of homogeneity of variance across groups. In addition, post hoc tests were performed using Tukey's honest significant difference to investigate significant differences among each level of the independent variable. A significant level was set at $\alpha=0.05$. 
FIGURE 2 Definitions of AOls. AOls, areas of interests
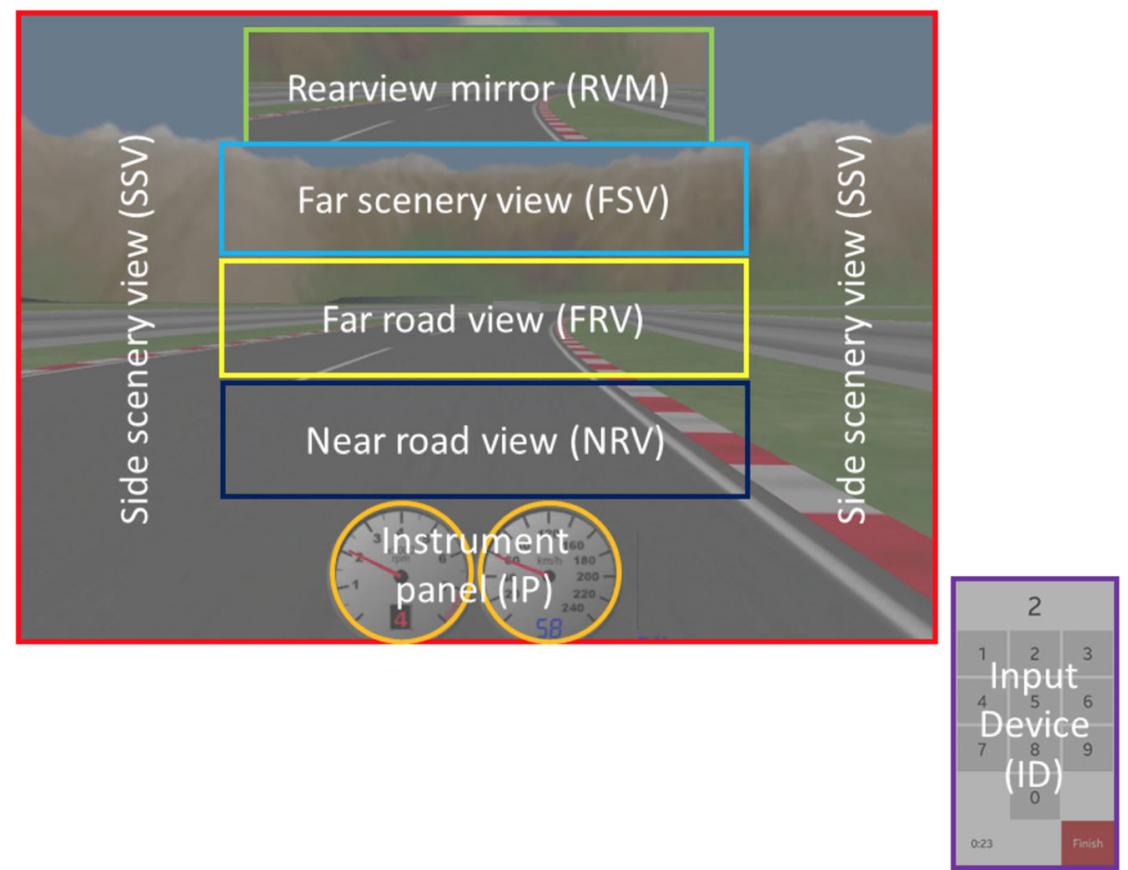

\section{RESULTS}

\subsection{Number of glances on each AOI}

Figure 3 shows the mean number of glances by each $\mathrm{AOI}$ and the secondary task type. The FRV, 39.5\% and ID, 31.4\% had the highest percentage of the number of glances.

A Kruskal-Wallis $\mathrm{H}$ test showed that there was a statistically significant difference in the number of glances at FRV between the different task types, $H(3)=21.73, p<0.001$. Pairwise comparisons showed A-S type led to the smaller number of glances at FRV than other three types of secondary task (in the sequence of $\mathrm{V}-\mathrm{S}>\mathrm{V}-\mathrm{M}>\mathrm{A}-\mathrm{M}>\mathrm{A}-\mathrm{S}$ ). For the number of glances at ID, it also showed a significant difference between the different task types in the same sequence $(H(3)=71.80, p<0.001)$. Posthoc pairwise comparisons revealed that the number of glances at ID for each type of secondary task differed significantly each other (all $p<0.05$ ), except between $\mathrm{V}-\mathrm{M}$ and $\mathrm{A}-\mathrm{M}$, and between $\mathrm{V}-\mathrm{M}$ and $\mathrm{V}$-S types.

No difference was found for the number of glances at both FRV and ID between different curves.

\section{2 | Total glance time on each AOI}

Figure 4 shows the mean total glance time by each $\mathrm{AOI}$ and the secondary task type. Similar to the number of glances measure, FRV (61.0\%) and ID (24.5\%) are the AOIs that accounted for the two highest portions of total glance time.

A statistically significant difference was revealed between the total glance time at FRV by different task types $(H(3)=47.02, p<0.001$. Posthoc comparison tests showed that the total glance time at FRV were all significantly different each other, except V-M and V-S types. There was also a significant difference in the total glance time at ID between the
FIGURE 3 Mean number of glances by each $\mathrm{AOI}$ and the secondary task type. Error bars represent standard errors of the mean. AOI, areas of interest
10.0

\section{- A-M $\boldsymbol{\Delta A}-\mathrm{S}$ 四-M EV-S}

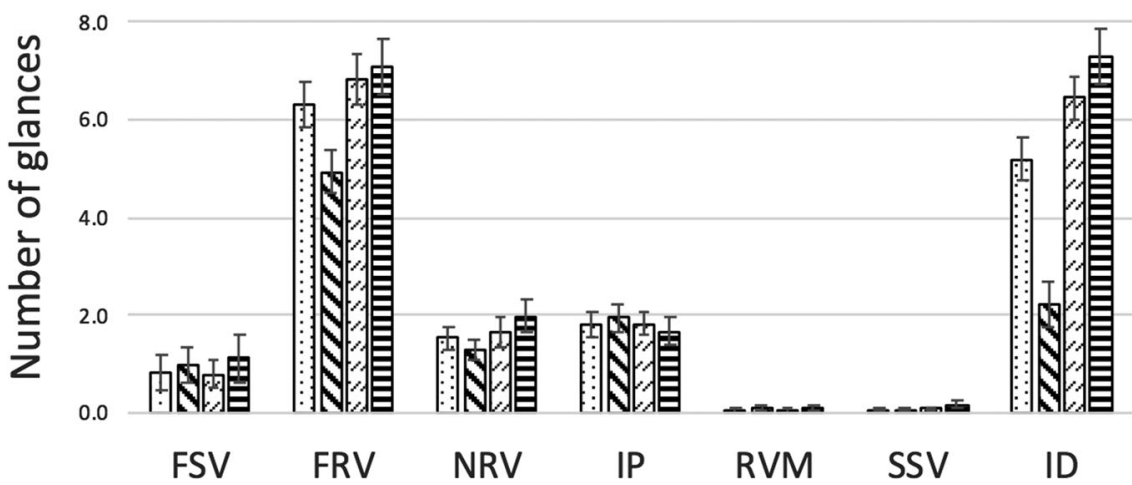




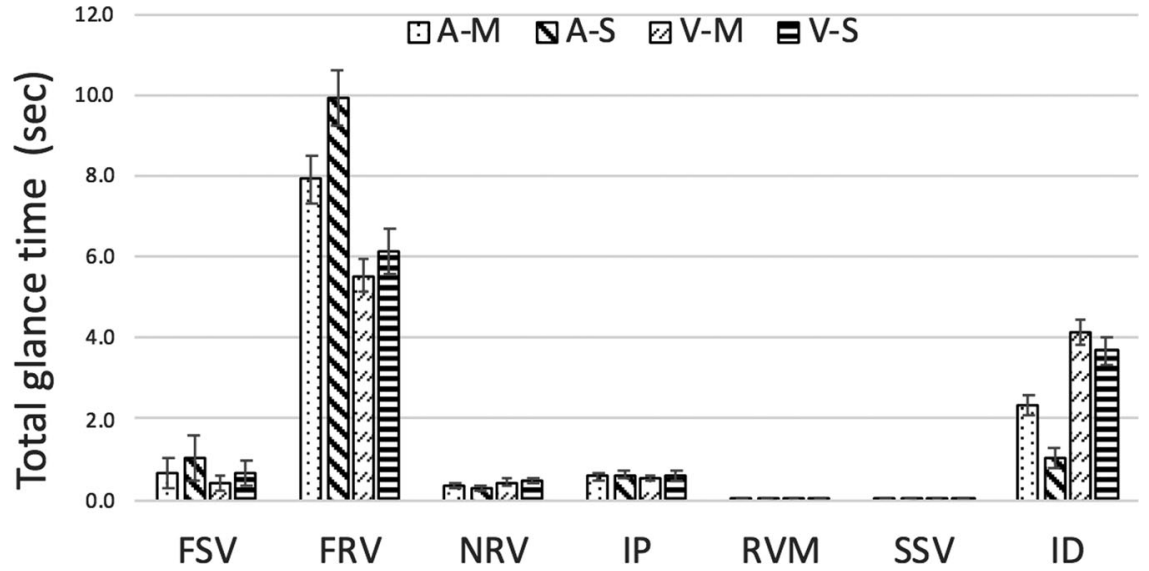

FIGURE 4 Mean total glance time by each $\mathrm{AOI}$ and the secondary task type. Error bars represent standard errors of the mean. AOI, areas of interest different task types, $H(3)=80.17, p<0.001$. Pairwise comparisons showed that the total glance times at ID were also all significantly different each other, except V-M and V-S types.

Mann-Whitney's $U$ test $(U=1770.0, p<0.001)$ revealed that the total glance time at FRV was significantly longer at the sharp curve $(\bar{x}=8.50)$ than the moderate curve $(6.25)$, as shown in Figure 5. On the other hand, the total glance time at ID was longer at the moderate curve (3.10) than the sharp curve (2.53): $U=2454.5, p<0.012$.

\subsection{Visual scanning patterns based on the transition probability}

Glance transitions and the probabilities by secondary task type and road curvature are illustrated in Figure 6, for only transitions whose transition probabilities are equal to and greater than $17 \%$ (called reliable transitions). Note that this threshold of the reliable transitions is only for the illustration in Figure 6 to avoid its complexity, but all transitions were analyzed in this study. Moreover, some AOls illustrated in Figure 2 (e.g., SSV, IP) were simplified in Figure 6. In general, drivers showed a fewer number of reliable transitions on the sharp curve than the moderate curve. Among the secondary task types, the number of reliable transitions was the

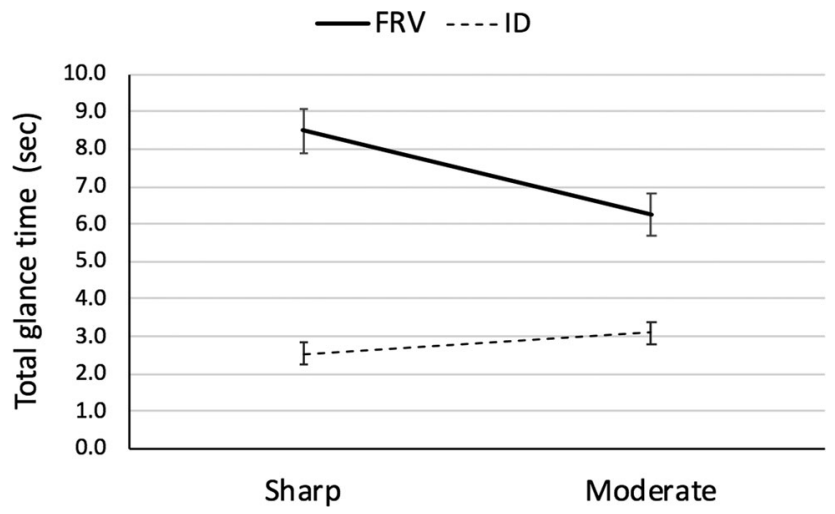

FIGURE 5 Mean total glance time at FRV and ID for the sharp and moderate curves. Error bars represent standard errors of the mean. FRV, far road view; ID, input device greatest when the V-S type was used, and the least when the A-M type was used.

One of the striking features of the transitions is that while driving on the sharp curve with the A-M task, all glances that were fixated in the rear-view mirror and the side scenery view transited into the far road view. The similar feature was also shown while driving on the sharp curve with the A-S task, but all fixations from the side scenery view went to near road view, not far road view.

Drivers showed the dominance of transitions to the far road view right before and after the eyes-off-road fixations (i.e., input device or instrument panel). This might be because drivers tend to secure a clear view for safe driving before and after looking at the input device or instrument panel. The highest probabilities of transitions between the far road view and the eyes-off-road fixations showed while driving on the sharp curve with the V-M secondary tasks.

\section{4 | Visual entropy}

Visual entropy was significantly higher while driving on the moderate curve than the sharp curve $(U=829.0, p<0.001)$, as shown in Figure 7. However, it was revealed that a nonsignificant effect of the secondary task type on the entropy $(U=3.05, p<0.38)$.

\section{5 | Visual stationary probabilities at FVR and ID}

The effects of curvedness (sharp vs. moderate) and the type of secondary tasks (A-M, A-S, V-M, and V-S) were tested as withinsubject factors, with respect to the visual stationary probabilities at two major AOls, FRV and ID. ANOVA revealed a statistically significant main effect of curvedness on the mean stationary probability at the $\operatorname{FRV}\left(F(1,152)=8.146, p=0.005, \eta_{p}{ }^{2}=.051\right)$ and $\operatorname{ID}\left(F(1,143)=6.194, p=0.014, \eta_{p}{ }^{2}=0.042\right)$. As shown in Figure 8 , the mean stationary probability at the FRV was significantly higher for the sharp curve $(M=42.2 \%, S D=9.1 \%)$ than moderate curve $(M=38.5 \%, S D=6.9 \%)$. Similarly, the visual stationary probability at the ID was significantly higher for the sharp curve $(M=35.1 \%$, $S D=10.1 \%)$ than the moderate curve ( $M=31.6 \%, S D=10.2 \%)$.

Results also showed a significant main effects of task type on the stationary probability at the $\operatorname{FRV}(F(3,152)=3.467, p=0.018$, 


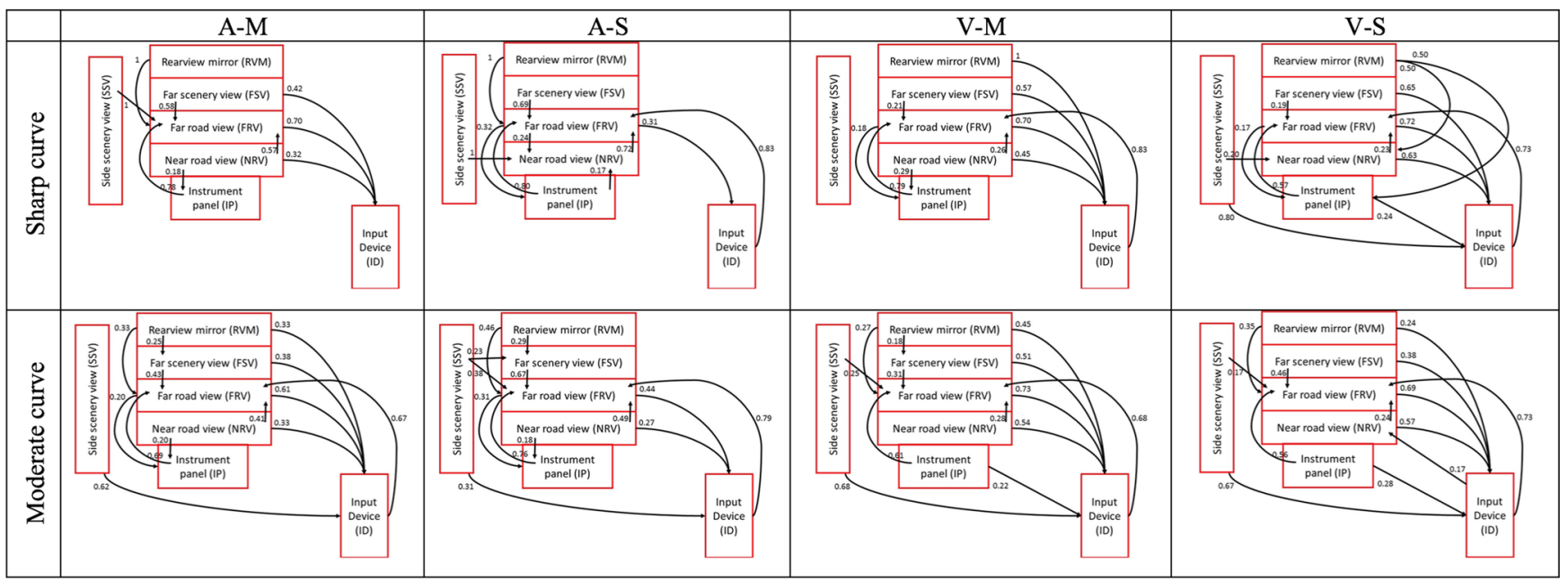

FIGURE 6 Glance transitions by road curvatures and secondary task types

$\mathbb{\$}$ Sharp $\square$ Moderate

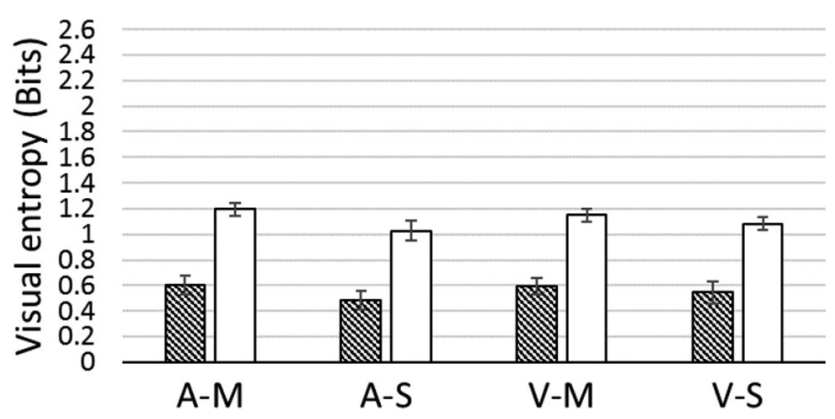

FIGURE 7 Mean visual entropy for the sharp and moderate curves in the different secondary task types. Error bars represent standard errors of the mean

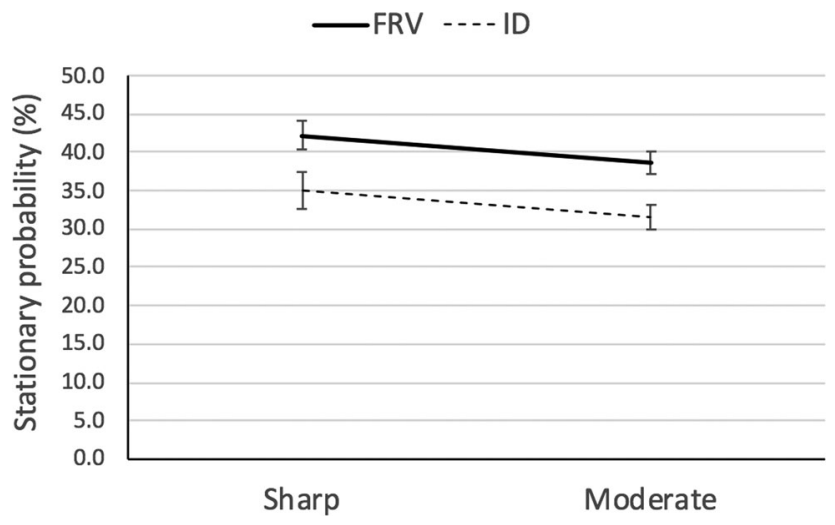

FIGURE 8 Visual stationary probabilities at FRV and ID for the sharp and moderate curves. Error bars represent standard errors of the mean. FRV, far road view; ID, input device

$\left.\eta_{p}{ }^{2}=0.064\right)$ and ID $\left(F(3,143)=26.689, p<0.001, \eta_{p}{ }^{2}=.359\right.$; see Figure 9). The mean stationary probabilities at FRV were highest for the A-S type $(M=43.8 \%, S D=6.3 \%)$ and the similarly lowest for the V-M $(M=39.1, S D=8.5 \%)$ and V-S types $(M=38.3 \%, S D=9.0 \%)$. Pairwise comparisons with Tukey correction revealed that only the two differences between A-S and V-M, and between A-S and V-S were significant. With respect to the mean stationary probabilities at the ID, they were highest for the $V-S$ type $(M=38.4 \%, S D=6.8 \%)$, followed by the $V-M$ type $(M=37.0 \%, S D=5.1 \%)$, the A-M $(M=32.7 \%, S D=8.0 \%)$, and the A-S type $(M=20.8 \%, S D=13.1 \%)$. Post hoc Tukey's tests revealed that the mean stationary probabilities at the ID for each secondary task type differed significantly from each other type (all $p<0.05$ ), except between $\mathrm{V}-\mathrm{M}$ and $\mathrm{A}-\mathrm{M}$, and between $\mathrm{V}-\mathrm{M}$ and $\mathrm{V}$-S types.

The analysis showed no significant interaction effect of curvedness and secondary task type on the visual stationary probabilities at both FVR and ID $(p>0.05)$.

\section{6 | Driving speed}

Figure 10 represents the distribution of driving speed by road curvature and secondary task type. The road curvature $(p<.001)$ and the secondary task type $(p<.001)$ had significant effects on driving speed. The driving speed was significantly higher in the moderate curvature $(M=71.1, S D=8.58)$ than the sharp curvature $(M=66.6$, $S D=8.64)$. Post hoc test showed clear significant differences among the 4 secondary task types: $A-S(M=72.8, S D=7.52)>A-M(M=71.4$, $S D=7.71)>V-S(M=70.1, S D=8.52)>V-M(M=68.0, S D=10.0)$.

\section{5 | DISCUSSION}

\section{1 | Effects of road curvature}

On sharper curves, drivers showed longer total glance times at FRV and a lower number of reliable transitions between AOls. They also had lower visual entropy, indicating that drivers on the sharper curves were likely to spend time monitoring particular areas. In other words, drivers are more likely to scan only a few areas of interest with a long duration in sharper curves, which is an unsafe driving behavior because they could not detect potential visual hazards. Previous studies revealed that drivers' visual demand (i.e., the 


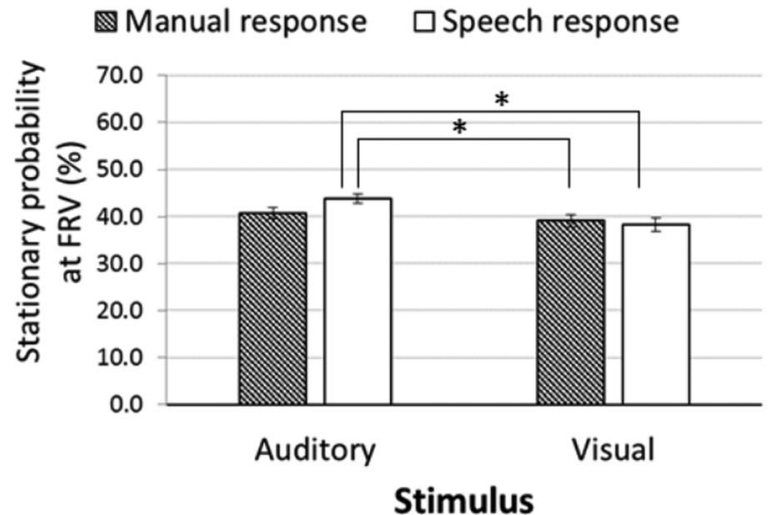

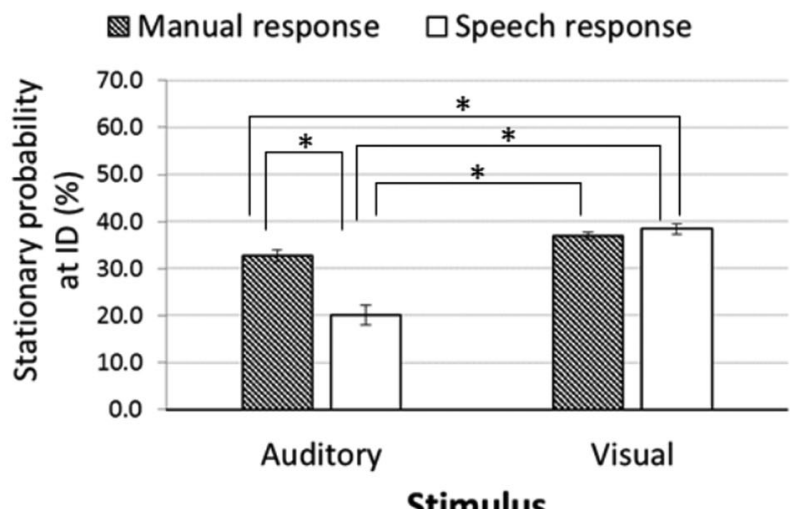

Stimulus

FIGURE 9 Visual stationary probabilities at FRV and ID in the different secondary task types. Error bars represent standard errors of the mean. FRV, far road view; ID, input device

percentage of time spent viewing the road) increases when they drive on sharper curves (Tsimhoni \& Green, 2001; Wooldridge, Fitzpatrick, Koppa, \& Bauer, 2000). The current study supports their results, as it was found that drivers tend to concentrate their visual attention more on the road at the sharper curves, probably to maintain the driving safety, given that the driving speed was significantly lower at the sharper curves.

Total glance time at ID, indicating total head-down glance time, was longer while driving on the moderate curve than the sharp curve. On the other hand, the visual stationary probability at ID was higher for the sharper curves, meaning that the probability of head-down glances is higher for the sharp curve after enough time has passed for sequences of driver eye fixation between each $\mathrm{AOI}$ reach the stationary distribution. Since the actual time when driving on the sharp curve was quite a short period of time (shorter than $30 \mathrm{~s}$ at most), there might not be enough information to determine on which areas of interest the drivers were most fixated.

\section{2 | Effects of secondary task type}

It was found that the number of reliable transitions between $\mathrm{AOls}$ was the greatest when the V-S type was used, and the least when the A-M type was used. The visual stimuli used in the experiment were Arabic numerals that appeared randomly every $3 \mathrm{~s}$. Drivers' eye fixations probably had to move between the input device and the curved road, potentially causing them to miss appearances of the stimuli. Thus, it might be difficult for drivers to identify visual stimuli on the input device while driving on curved roads. On the other hand, since the buttons on the input device used for the manual method were in a fixed location, it might be relatively easy for drivers to locate the buttons, leading to fewer numbers of transitions between AOIs.

The total glance time at FRV was longest when the A-S type of secondary task (the less-visually demanding task) was performed, followed by A-M, V-S, and V-M. It was found that this sequence is consistent with the sequence of driving speed participants drove during the four sessions. This finding can imply that the driving speed can be a predictor for drivers' eye movements, especially for how long drivers look at the far road view.

A statistical test revealed that the secondary task type has a significant effect on the number of glances at FRV: the number of glances at FRV was the smallest when the A-S type of secondary task was performed. In other words, while performing relatively less-visually demanding tasks (compared with visual or manual tasks), drivers looked (a)

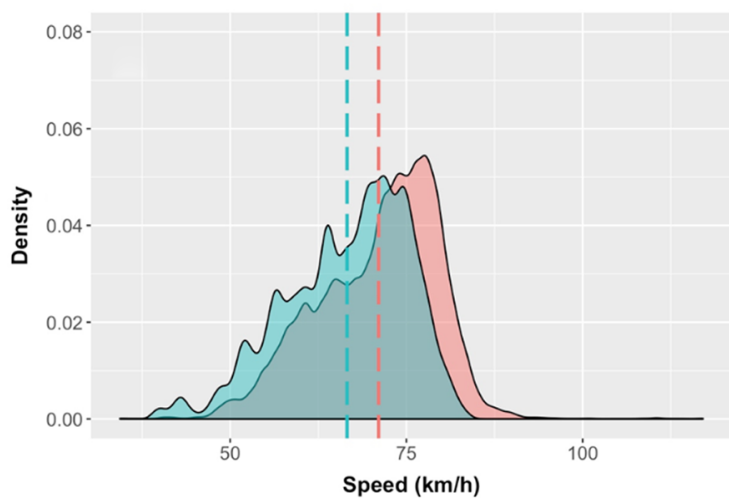

(b)

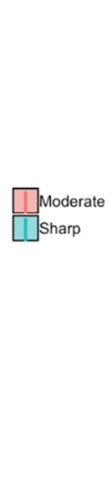

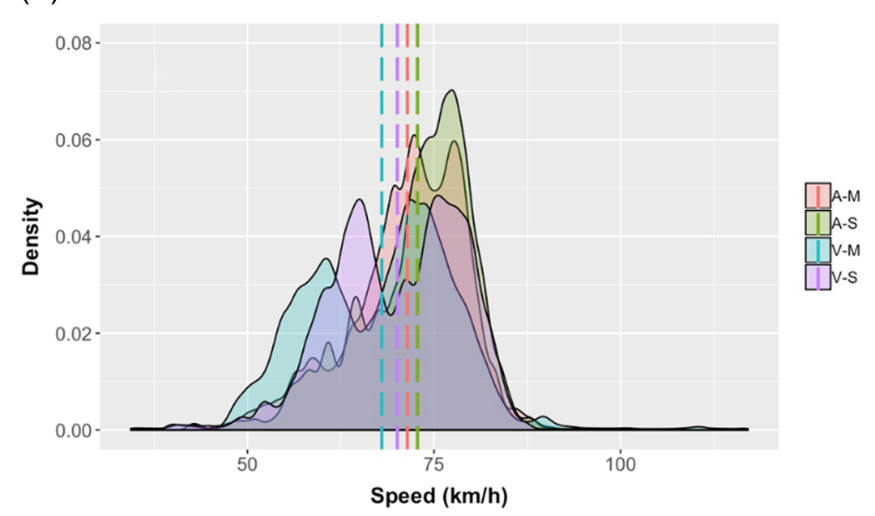

FIGURE 10 Distribution of driving speed (a) by road curvature and (b) by secondary task type. Horizontal lines represent the mean of speed for each condition 
at the FRV less frequently. On the other hand, the visual stationary probabilities at FRV were higher when drivers used A-S type of tasks (than V-M and V-S types of tasks), meaning that total expected time spent at FRV is higher for the A-S type, once sequences of driver eye fixation between the each $\mathrm{AOI}$ reach the stationary distribution. These inconsistent results might be because drivers in this experiment were able to secure more spare time spent focusing other places than the FRV while doing the A-S task, thus were less focused on looking at the road in front of them. However, in a long-term examination of visual scanning, the probabilities of a glance staying at FRV is higher for the A-S type of task, which seems reasonable.

A higher visual entropy indicates higher randomness or higher visual scanning complexity, being likely to scan more areas with a shorter period of time in each area (Wang et al., 2017). The analysis of visual entropy revealed no significant difference among the 4 secondary task types. In other words, no significant difference among the secondary task's modality type was shown in regard to how random drivers' scanning pattern is; specifically, how more or fewer areas drivers scan, how shorter or longer period of time drivers' glance are fixated on each area.

\section{3 | Value of markov analysis in visual scanning}

Markov analysis can contribute many unique insights into drivers' glance behaviors and scanning patterns. Measures of dynamic eye movements, using the distributions and probabilities of eye fixation and duration, can provide insights to better understand underlying cognitive processes and scanning strategies while driving and performing secondary tasks, compared with conventional eye movement measures, such as the frequency and duration of fixations on AOls. First, visual transition probability can be used as a measure when the transition between two fixations is more prevalent than other transitions, which typical static measures cannot inspect. If there is a more dominant transition between specific AOls over others, it may indicate that the drivers' visual attention shifts due to mental processes relating to their internal expectations and/ or external environment. A greater number of transitions between particular AOIs likely indicates completion of secondary tasks (i.e., internal) and/or driving hazards on curves (i.e., external). Second, visual entropy can illustrate an individual's visual scanning complexity; how balanced drivers scan the possible viewing areas of interest during particular tasks. Entropy can also be used to diagnose an individual's emotional status, evidenced by pilots' increased visual entropy when they have higher cognitive anxiety (Allsop \& Gray, 2014). Third, the analysis of visual stationary probability enables estimation of the proportion of time spent fixating on specific AOIs in the long run, which is a useful indicator of driving safety, especially in the long-run repeated situations.

\section{4 | Limitations and suggestions for future research}

We are aware that our research may have two limitations. First, the effect of curve direction was not considered in this study. Drivers' glance behavior may vary depending on the direction of curved roads because the location of the input device was fixed on the right side of the driver while the roads curved both left and right. This study focused on eye scanning patterns related to driving safety, thus road curvature (rather than curve direction) was chosen as an independent variable, based upon the literature. For example, Milton and Mannering (1998) revealed that a decrease in curve radii increased the frequency in the number of car crashes. Further work needs to be carried out to investigate whether the curve direction affects the driver glance behavior. We believe that our research will serve as a base for future study.

Second, the current study focused little on individual differences, although visual scanning patterns vary by individuals (Kang \& Landry, 2015; Noton \& Stark, 1971). While we focused primarily on external factors (i.e., road curvatures and secondary tasks) that may affect drivers' glance behavior, we observed some instances of abnormal cognitive processes: several participants showed quite a high number of glances (S22, S23) and long total glance time (S21, S24) at the far road view when driving on moderate curves while performing speech tasks (i.e., A-S and V-S). Their long total glance time (longer than $100 \mathrm{~s}$ ) at the far road view may indicate the mind wandering, based upon the conclusion of Reichle, Reineberg, and Schooler (2010) that fixation duration is longer while reading mindlessly than while reading normally. The high number of glances (more than 70 times) at the far road view may represent frequent eye movements from the far road view to other areas of interest, and unnecessary visual attention on the road due to the less-visually demanding speech tasks. Future work will explore the individual differences, such as comparing novice and experienced drivers (e.g., Crundall \& Underwood, 1998; Pradhan et al., 2005; Underwood et al., 2003) or age difference (e.g., Bao \& Boyle, 2009; Rodrick, Bhise, \& Jothi, 2013; Schieber \& Gilland, 2008) while driving on a curved road with different types of secondary tasks.

\section{ACKNOWLEDGMENTS}

This study was supported by funding from a Rackham Graduate Student Research Grant from the University of Michigan [grant number U051990]. The authors are grateful to Hideaki Nakao from the University of Michigan who provided initial insights in data analysis. We would also like to thank the anonymous reviewers of the article for their valuable feedback.

\section{ORCID}

Heejin Jeong (D) http://orcid.org/0000-0002-0122-532X

\section{REFERENCES}

Allsop, J., \& Gray, R. (2014). Flying under pressure: Effects of anxiety on attention and gaze behavior in aviation. Journal of Applied Research in Memory and Cognition, 3(2), 63-71.

Bao, S., \& Boyle, L. N. (2009). Age-related differences in visual scanning at median-divided highway intersections in rural areas. Accident Analysis \& Prevention, 41(1), 146-152. 
Crundall, D. E., \& Underwood, G. (1998). Effects of experience and processing demands on visual information acquisition in drivers. Ergonomics, 41(4), 448-458.

Ellis, S. R., \& Stark, L. (1986). Statistical dependency in visual scanning. Human Factors: The Journal of the Human Factors and Ergonomics Society, 28(4), 421-438.

Green, P. (2015). Where do drivers look while driving (and for how long). Human factors in traffic safety, 77-110.

Harris Sr, R. L., Glover, B. J., \& Spady Jr, A. A. (1986). Analytical techniques of pilot scanning behavior and their application (NASA Tech. Rep. No. 2525). Hampton, VA: Langley Research Center.

Haslbeck, A., \& Zhang, B. (2017). I spy with my little eye: Analysis of airline pilots' gaze patterns in a manual instrument flight scenario. Applied Ergonomics, 63, 62-71.

Hillier, F. S. (2012). Introduction to operations research. Tata McGraw-Hill Education.

International Organization for Standardization. (2002). ISO 15007- 1: Road vehicles-Measurement of driver visual behavior with respect to transport information and control systems-Part 1: Definitions and parameters. Retrieved from http://www.iso.org

Itoh, Y., Hayashi, Y., Tsukui, I., \& Saito, S. (1990). The ergonomic evaluation of eye movement and mental workload in aircraft pilots. Ergonomics, 33(6), 719-732.

Jeong, H., \& Liu, Y. (2019). Effects of non-driving-related-task modality and road geometry on eye movements, lane-keeping performance, and workload while driving. Transportation Research Part F: Psychology and Behaviour, 60, 157-171.

Kang, Z., \& Landry, S. J. (2014). Using scanpaths as a learning method for a conflict detection task of multiple target tracking, 56(6), 1150-1162.

Kang, Z., \& Landry, S. J. (2015). An eye movement analysis algorithm for a multielement target tracking task: Maximum transition-based agglomerative hierarchical clustering. IEEE Transactions on Human-Machine Systems, 45(1), 13-24.

Krejtz, K., Duchowski, A., Szmidt, T., Krejtz, I., González Perilli, F., Pires, A., ... Villalobos, N. (2015). Gaze transition entropy. ACM Transactions on Applied Perception (TAP), 13(1), 4-20.

Liang, Y., Horrey, W. J., \& Hoffman, J. D. (2015). Reading text while driving: Understanding drivers' strategic and tactical adaptation to distraction. Human Factors: The Journal of the Human Factors and Ergonomics Society, 57(2), 347-359.

Liang, Y., Lee, J. D., \& Yekhshatyan, L. (2012). How dangerous is looking away from the road? Algorithms predict crash risk from glance patterns in naturalistic driving. Human Factors: The Journal of the Human Factors and Ergonomics Society, 54(6), 1104-1116.

Liechty, J., Pieters, R., \& Wedel, M. (2003). Global and local covert visual attention: Evidence from a Bayesian hidden Markov model. Psychometrika, 68(4), 519-541.

Marchitto, M., Di Stasi, L. L., \& Cañas, J. J. (2012). Ocular movements under taskload manipulations: Influence of geometry on saccades in air traffic control simulated tasks. Human Factors and Ergonomics in Manufacturing \& Service Industries, 22(5), 407-419.

Milton, J., \& Mannering, F. (1998). The relationship among highway geometrics, traffic-related elements and motor-vehicle accident frequencies. Transportation, 25(4), 395-413.

Mourant, R. R., \& Rockwell, T. H. (1970). Mapping eye-movement patterns to the visual scene in driving: An exploratory study. Human Factors: The Journal of the Human Factors and Ergonomics Society, 12(1), 81-87.

Noton, D., \& Stark, L. (1971). Eye movements and visual perception. Scientific American, 224(6), 34-43.

Pradhan, A. K., Hammel, K. R., DeRamus, R., Pollatsek, A., Noyce, D. A., \& Fisher, D. L. (2005). Using eye movements to evaluate effects of driver age on risk perception in a driving simulator. Human Factors: The Journal of the Human Factors and Ergonomics Society, 47(4), 840-852.
Reichle, E. D., Reineberg, A. E., \& Schooler, J. W. (2010). Eye movements during mindless reading. Psychological Science, 21(9), 1300-1310.

Reimer, B., Gulash, C., Mehler, B., Foley, J. P., Arredondo, S., \& Waldmann, A. (2014). The MIT AgeLab n-back: a multi-modal android application implementation, Adjunct Proceedings of the $6^{\text {th }}$ International Conference on Automotive User Interfaces and Interactive Vehicular Applications. New York, NY: ACM. (pp. 1-6)

Robinson, G. H., Erickson, D. J., Thurston, G. L., \& Clark, R. L. (1972). Visual search by automobile drivers. Human Factors: The Journal of the Human Factors and Ergonomics Society, 14(4), 315-323.

Rodrick, D., Bhise, V., \& Jothi, V. (2013). Effects of driver and secondary task characteristics on lane change test performance. Human Factors and Ergonomics in Manufacturing \& Service Industries, 23(6), 560-572.

Ross, S. M. (2014). Introduction to probability models. Academic press.

Schieber, F., \& Gilland, J. (2008). Visual Entropy metric reveals differences in drivers' eye gaze complexity across variations in age and subsidiary task load. Proceedings of the Human Factors and Ergonomics Society Annual Meeting, 52(23), 1883-1887.

Shannon, C., \& Weaver, W. (1998). The Mathematical Theory of Communication. Urbana: University of Illinois Press.

Sivak, M. (1996). The information that drivers use: Is it indeed $90 \%$ visual? Perception, 25(9), 1081-1089.

Society of Automotive Engineers (2000, July). Definition and Experimental Measures Related to the Specification of Driver Visual Behavior Using Video Based Techniques (SAE Recommended Practice J2396, Warrendale, PA: Society of Automotive Engineers.

Sodhi, M., Reimer, B., \& Llamazares, I. (2002). Glance analysis of driver eye movements to evaluate distraction. Behavior Research Methods, 34(4), 529-538.

di Stasi, L. L., Diaz-Piedra, C., Rieiro, H., Carrión, J. M. S., Berrido, M. M., Olivares, G., \& Catena, A. (2016). Gaze entropy reflects surgical task load. Surgical Endoscopy, 30(11), 5034-5043.

Tsimhoni, O., \& Green, P. (2001). Visual demand of driving and the execution of display-intensive in-vehicle tasks, In Proceedings of the Human Factors and Ergonomics Society Annual Meeting (45, 23, 1586-1590). Los Angeles, CA: AGE Publications.

Underwood, G., Chapman, P., Brocklehurst, N., Underwood, J., \& Crundall, D. (2003). Visual attention while driving: Sequences of eye fixations made by experienced and novice drivers. Ergonomics, 46(6), 629-646.

Victor, T., Dozza, M., Bärgman, J., Boda, C. N., Engström, J., Flannagan, C., ... Markkula, G. (2015). Analysis of naturalistic driving study data: Safer glances, driver inattention, and crash risk (No. SHRP 2 Report S2-S08ARW-1).

Wang, Y., Bao, S., Du, W., Ye, Z., \& Sayer, J. R. (2017). Examining drivers' eye glance patterns during distracted driving: Insights from scanning randomness and glance transition matrix. Journal of Safety Research, 63, 149-155.

Werneke, J., \& Vollrath, M. (2012). What does the driver look at? The influence of intersection characteristics on attention allocation and driving behavior. Accident Analysis \& Prevention, 45, 610-619.

Wooldridge, M., Fitzpatrick, K., Koppa, R., \& Bauer, K. (2000). Effects of horizontal curvature on driver visual demand. Transportation Research Record: Journal of the Transportation Research Board, (1737), 71-77.

How to cite this article: Jeong $\mathrm{H}$, Kang Z, Liu Y. Driver glance behaviors and scanning patterns: Applying static and dynamic glance measures to the analysis of curve driving with secondary tasks. Hum. Factors Man. 2019;29:437-446. https://doi.org/10.1002/hfm.20798 